\title{
Dog and owner demographic characteristics and dog personality trait associations
}

\author{
Enikő Kubinyi* ${ }^{*}$, Borbála Turcsán ${ }^{1}$, Ádám Miklósi ${ }^{1}$ \\ Eötvös University, Department of Ethology, H-1117, Pazmany P. s. 1/c, Budapest, Hungary
}

\section{A R T I C L E I N F O}

\section{Article history:}

Received 9 December 2008

Received in revised form 8 April 2009

Accepted 8 April 2009

\section{Keywords:}

Personality

Demography

Dog

Owner

Regression tree

\begin{abstract}
A B S T R A C T
The aim of this study was to analyze the relationships between four personality traits (calmness, trainability, dog sociability and boldness) of dogs (Canis familiaris) and dog and owner demographics on a large sample size with 14,004 individuals. German speaking dog owners could characterize their dog by filling out a form on the Internet. There were five demographic variables for dogs and nine for owners. Two statistical methods were used for investigating the associations between personality and demographic traits: the more traditional general linear methods and regression trees that are ideal for analyzing nonlinear relationships in the structure of the data. The results showed that calmness is influenced primarily by the dog's age, the neutered status, the number of different types of professional training courses (e.g. obedience, agility) the dog had experienced and the age of acquisition. The least calm dogs were less than 2.5 years old, neutered and acquired after the first 12 weeks of age, while the calmest dogs were older than 6.9 years. Trainability was affected primarily by the training experiences, the dog's age, and the purpose of keeping the dog. The least trainable dogs had not received professional training at all and were older than 3 years. The most trainable dogs were those who participated in three or more types of professional training. Sociability toward conspecifics was mainly determined by the age, sex, training experience and time spent together. The least sociable dogs were older than 4.8 years and the owners spent less than $3 \mathrm{~h}$ with the dog daily. The most sociable dogs were less than 1.5 years old. Males were less sociable toward their conspecifics than females. Boldness was affected by the sex and age of the dog and the age of acquisition. The least bold were females acquired after the age of 1 year or bred by the owner. The boldest dogs were males, acquired before the age of 12 weeks, and were younger than 2 years old. Other variables, including the owner's gender, age, education, previous experience with dogs, the number of people and dogs in the household, and purpose of keeping the dogs had minor, but detectable effects. The results suggest that on-line questionnaires are a very effective means for collecting data about dog behavior, especially if owners are motivated by instant feedback. However, note that the characteristics of dogs in the present study were reported by the owners, and the associations with the traits do not necessarily represent a causal relationship.
\end{abstract}

(c) 2009 Elsevier B.V. All rights reserved.

\section{Introduction}

Personality is often defined as an individual's distinctive pattern of behavior (besides feeling and thinking) that is consistent across time and situations (e.g. Pervin and John, 1997). Personality studies in dogs have become very popular in the last decade. With the keywords "dog" and "personality or temperament," Jones and Gosling (2005) found 51 references from science databases published between 1934 and 2004. In November 2008, according to our literature search in the Web of Knowledge,

\footnotetext{
* Corresponding author. Tel.: +36 1 3812179; fax: +36 13812180 .

E-mail addresses: kubinyie@gmail.com (E. Kubinyi), turcsanbori@gmail.com

(B. Turcsán), amiklosi62@gmail.com (Á. Miklósi).

${ }^{1}$ Tel.: +361 3812179; fax: +3613812180.
}

this number had increased with at least 30 recent publications. Extensive reviews have also been published recently (e.g. Jones and Gosling, 2005; Diederich and Giffroy, 2006). This indicates that dog personality is a matter of great public concern, and besides theoretical interest, it has a wide range of practical applications, including significant influence on the dog-human bond.

So far, personality research has focused on (1) developing tools for characterizing behavior (e.g. Sheppard and Mills, 2002; Hsu and Serpell, 2003; Ley et al., 2008), (2) looking at breed (genetic) differences (e.g. Wilsson and Sundgren, 1997; van Oers et al., 2005; Svartberg, 2002, 2006; Strandberg et al., 2005), and (3) studying the effect of development or stability of the behavior characteristics over an extended time. In the latter case, individuals are repeatedly tested in early puppyhood, at a juvenile age (time of sexual maturation) and later in adulthood with the aim of evaluat- 
ing the predictability of certain early behavioral characteristics (e.g. Wilsson and Sundgren, 1998; Slabbert and Odendaal, 1999).

Importantly, however, as Jones and Gosling (2005) pointed out, the vast majority of dogs tested were in working contexts, and pet dogs, with a fuller representation of dog breeds, were relatively neglected. Also, they mention that few studies investigated dogs over the age of 4 years, so we know little about how aging affects personality traits. The situation is similar with neutering, although previous studies suggest that there are several personality differences between intact and neutered dogs (e.g. Podberscek and Serpell, 1996). Recent studies have started to compensate for this imperfection and investigated the associations between dog behavior and independent variables. For example, Bennett and Rohlf (2007) studied the relationship between demographic variables (several were unusual, like the amount of experience the owner reported having with dogs, owners' age, family size, etc.) and dog behavior with a questionnaire survey in 413 adult individuals. They found that problematic behaviors were associated with numerous owner and dog characteristics, although most differences were small. For example, the number of people in the household positively correlated with aggression and disobedience. Dogs acquired from a pet shop had more problematic behaviors. However, involvement in professional training courses and other shared activities decreased the occurrence of problematic behaviors (see also Kobelt et al., 2003; Jagoe and Serpell, 1996 for similar results, both based on questionnaires, with several relatively rarely observed characteristics).

However, surveying problematic behaviors exposes only a facet of dog personality. People who would voluntarily complete a questionnaire about their dogs' behavior experience relatively few problematic behaviors (Bennett and Rohlf, 2007). The interaction of demographic variables in a large sample of dog owners might reveal yet uncovered associations.

In this study, we aimed to reveal associations between dog personality traits and both dog and owner demographic variables in a large sample size. Demographic variables were chosen mainly based on previously described effects in the literature, like dog's age and sex (in test batteries: see Wilsson and Sundgren, 1997; Seksel et al., 1999; Strandberg et al., 2005; Saetre et al., 2006; Svartberg, 2006; in questionnaire-based ratings of individual dogs: Jagoe and Serpell, 1996; Hsu and Serpell, 2003; Rooney and Bradshaw, 2004; Ley and Bennett, 2008), but some sparsely investigated variables were also introduced, like the dog's age at acquisition, number of people and dogs in the household, owner's experience with previous dogs, purpose of keeping the dog and time investment in the dog.

In the present study, we used the Internet as the medium for reaching a large audience within a short time period. Our sample is representative of people who are relatively engaged with their dog: they are probably reading dog magazines, are interested in the popular dog literature and are keen on learning some new aspects of their dog's personality. Accordingly, the present sample is a sub-population of German speaking dog owners, but could be considered to resemble dog owners in Western Europe with similar socioeconomic status. External validity of the present questionnaire was investigated by looking at reports available in the literature on dog personality and behavior.

\section{Method}

\subsection{Subjects}

In this study we collected owner's reports on 14,004 dogs. Owners filled in an on-line questionnaire in German which was advertised in the "Dogs" magazine (published by Living at Home Multi Media GmbH, Hamburg, August 2007 issue) and the magazine's website (www.dogs-magazin.de). It was accompanied by a short article and was available from the end of August 2007 to the beginning of January 2008. The first questionnaire arrived on August 28, 2007 and the last one on January 8, 2008.

Dogs younger than 1 year old (26.8\%) were excluded from this sample, because literature suggests that their behavior cannot be considered as stable over time. After this correction, the sample size was 10,519. The sample comprised 267 breeds which were represented by 1-527 individuals per breed (the latter was the Labrador Retriever). 3920 dogs were categorized by their owners as mixed-breed. The descriptive statistics of the sample are presented in Table 1 . The mean age $( \pm$ S.D.) of the dog in the sample was $4.2 \pm 3.1$ years. $56.1 \%$ of the dogs were males. $43.1 \%$ of the dogs were neutered (39\% of males and $48 \%$ of females). Half of the dogs were acquired before the age of 12 weeks (53.7\%), and only $1.9 \%$ were bred by the owner. Approximately one-third of the dogs (35.3\%) had not participated in any kind of professional training courses. The respondents were mainly female (79.6\%). The majority of them (64.9\%) were between 31 and 60 years old, and only 5.3\% were younger than 18 years old. Most of the respondents (40.3\%) had secondary education, $26.0 \%$ had high school, $22.3 \%$ had primary school and $11.4 \%$ had a university degree. The average number of people in the households ( \pm S.D.) was $2.8 \pm 1.4$. The majority $(41.7 \%)$ of the respondents resided in a two-person household. Participants reported owning only one dog $(66.9 \%)$ or two dogs (20.6\%). Family member $(93.3 \%)$ was marked as the most common purpose of keeping the dog. Other, non-exclusive functions were mentioned less frequently (hobby: $49.5 \%$; protection: $10.1 \%$; other work: $2.0 \%$, breeding: $2.4 \%$ ). $66.8 \%$ of the respondents had experience in keeping a dog previously (the mean ( \pm S.D.) number of previous dogs was $1.2 \pm 2.2$ ). Approximately $70 \%$ of the respondents claimed spending more than $3 \mathrm{~h}$ with the dog per day and playing with it every day.

\subsection{Procedure}

Dog owners were asked to complete two different questionnaires.

1. The "Demography Questionnaire" inquired about demographic attributes of the dog and the owner and social attributes of their interactions (Table 2).

2. The "Personality Questionnaire" was based on a 48item Human Personality Inventory which is available at de.outofservice.com/bigfive/ (Copyright: John, 2000) and adapted for dog behavior (Table 2 ).

42 owners were asked to complete the questionnaire a second time, 1 day after the first completion.

\subsection{Statistical analysis}

SPSS 13.0 was used for the analysis. To condense the items of the Personality Questionnaire, principal component analysis was used with Varimax rotation with Eigenvalue $>1$ (Kline, 1994). The number of extracted factors was decided after visual inspection, using the rules of the Scree test (Cattell, 1966). Factor scores were calculated automatically by the SPSS software using the Regression method. Cronbach's alpha was calculated to assess the internal reliability of extracted factors and for testing the repeatability of the questionnaire (DeVellis, 1991). Univariate general linear model tested the main and all two-way interaction effects of independent variables on the personality traits. Dog age, number of people in 
Table 1

Descriptive statistics of the dogs older than 1 year in the present study $(N=10,519)$.

\begin{tabular}{|c|c|c|}
\hline \multicolumn{2}{|l|}{ Dogs } & \multirow{2}{*}{$\frac{\text { Missing data }}{0}$} \\
\hline Age & Mean \pm S.D. $=4.2 \pm 3.1$ & \\
\hline Sex & Male: $56.1 \%$; female: $43.9 \%$ & 0 \\
\hline Neutered status & Intact: $56.9 \%$; neutered: $43.1 \%$ & 0 \\
\hline Age at acquisition & Bred by the owner: $1.9 \%$; $2-12$ weeks: $53.7 \%$; $3-12$ months: $22.6 \%$; >1 year: $21.7 \%$ & 0 \\
\hline Training experience & Nothing: 35.3\%; 1 type: 23.3\%; 2 types: $21.5 \%$; 3 types: $11.6 \%$; 4 or more types: $8.3 \%$ & 1 \\
\hline \multicolumn{2}{|l|}{ Owners } & Missing data \\
\hline Gender & Man: $20.4 \%$, woman: $79.6 \%$ & 1 \\
\hline Age & $<18$ years: $5.3 \% ; 19-30$ years: $26.9 \%, 31-60$ years: $64.9 \%,>60$ years: $2.9 \%$ & 1 \\
\hline Education & Primary school: $22.3 \%$; secondary school: $40.3 \%$; high school: $26.0 \%$; college degree: $11.4 \%$ & 1 \\
\hline Number of people in the household & Mean \pm S.D. $=2.8 \pm 1.4$ & 10 \\
\hline Number of other dogs in the household & 0: $66.9 \% ; 1: 20.6 \% ; 2: 7.7 \% ;>2: 4.8 \%$ & 1 \\
\hline Purpose of keeping the dog & Family member exclusively: $45.1 \%$; family member + other: $48.2 \%$; not family member: $6.7 \%$ & 1 \\
\hline Number of previous dogs & Mean \pm S.D. $=1.2 \pm 2.2$ & 19 \\
\hline Hours spent with the dog per day & $0-1: 3.2 \% ; 1-3: 27.0 \% ;>3: 69.8 \%$ & 1 \\
\hline Frequency of playing with the dog per week & 1: 3.3\%; 2-3: 9.5\%; 4-5: 10.6\%; 6-7: 76.6\% & 1 \\
\hline
\end{tabular}

the household and number of previous dogs were covariates, sex of the dog, neutered status, age at acquisition, training experience, gender of the owner, age of the owner, education of the owner, number of other dogs in the household, purpose of keeping the dog, hours spent with the dog per day, frequency of playing with the dog per week were fixed factors. We carried out four regression tree analyses, one for each personality trait, to examine the relation between the demographic variables and the traits. Regression trees are ideal for analyzing complex numeric and/or categorical data and detecting non-linear relationships in the structure of the data (Karels et al., 2004; Low et al., 2006). We decided to use this method, because the large number of explanatory variables used in this study does not facilitate the revealing of complex interactions by the generally used univariate analyses. The tree is constructed by dividing data into mutually exclusive groups, called nodes. In one node, individuals have similar values for the dependent variable. The output is a tree diagram with a parent node at the top containing the entire data set. The parent node is split into child nodes based on the independent variable that reduces the most total variation within the dependent variable. Having considered all possible splits, the most suitable split is retained. The process is repeated on the next grouping level. The number of data divisions is determined using a cross-validation procedure by randomly drawing samples from the data set to evaluate the predictive error of the tree (De'Ath and Fabricius, 2000). According to Yamauchi et al. (2001), the resulting tree model resembles a human judgment process.

We used the CHAID statistical technique (Kass, 1980). CHAID uses an $F$ test if the variable is continuous (e.g. the dog's age in our case) and $\chi^{2}$ if the variable is categorical (e.g. gender of the owner). In order to facilitate interpretation, we specified the mini- mum number of cases as 2000 for parent nodes and 1000 for child nodes.

\section{Results}

\subsection{Factor extracting}

17 of the 24 items were grouped into four factors that accounted for $58 \%$ of the common variance in item scores. The stability of the factors was tested on a derived sample. We have randomly chosen 25 individuals from each breed with at least 25 representatives. Principal component analysis was run on this sample with the same parameter setting. Factor structure and the item-loadings were exactly the same as on the original sample that confirmed the stability of the factors (see Svartberg and Forkman, 2002).

Items loading higher than 0.5 were used for the interpretation of the factors. The factors were given the following labels: calmness (five items), trainability (five items), dog sociability (sociability toward dogs) (four items), and boldness (three items). Three out of the four Cronbach's alpha values were above 0.7, but the value for the boldness factor was lower (0.65), indicating that more related items would need to be added (Table 3 ). However, values above 0.6 are usually considered as satisfactory (e.g. Hsu and Serpell, 2003).

\subsection{Test-retest reliability}

The test-retest reliability of the factor was found to be acceptable: Cronbach's alpha for the factor scores of the first and second questionnaire-filling was 0.88 for calmness, 0.87 for trainability and sociability and 0.83 for boldness.

\section{Table 2}

Questionnaires applied in the study.

1. Demography questionnaire (dog and owner's characteristics)

Dog's name, breed, age, sex (male, female), neutered status (intact, neutered), age at acquisition (bred by the owner, 2-12 weeks, 3-13 months, >1 year), training experience (nothing, puppy class, basic class, obedience, assistance dog, guarding, agility, other).

Owner's gender (man, woman), age ( $<18,19-30,31-60,>60$ years), education (primary school, secondary school, high school, college degree); number of people in the household; number of other dogs in the household $(0,1,2,>2$ dogs); purpose of keeping the dog (family member, hobby, guarding, work, breeding); number of previous dogs; hours spent with the dog per day $(<1,1-3,>3 \mathrm{~h}$ ); frequency of playing with the dog per week (once, $2-3,4-5,6-7$ times).

2. Personality questionnaire (score $0-2$ )

The $\operatorname{dog}(1)$ is ingenious, inventive when seeks hidden food or toy; (2) is sometimes distressed, desolate; (3) is calm, even in ambiguous situations; (4) fights with conspecifics frequently; (5) is active, eager; (6) is stubborn, energetic; (7) can be stressed easily; (8) is ready to share toys with conspecifics; (9) is intelligent, learns quickly; (10) is rather cool, reserved; (11) is shy with conspecifics; (12) is not hostile with people; (13) is very easy to warm up to a new toy; (14) is unassertive, aloof when unfamiliar persons enter the home; (15) is emotionally balanced, not easy to rile; (16) is passionless and holds him/herself apart; (17) often does not understand what was expected from him/her during playing; (18) is sometimes fearful, awkward; (19) is cool-headed even in stressful situations; (20) is bullying with conspecifics; (21) is not much interested except in eating and sleeping; (22) is very self-confident; (23) is sometimes anxious and uncertain; (24) gets on well with conspecifics. 
Table 3

Factor structure, loadings of items, explained variance, Cronbach's alpha and Eigenvalues of factors. Loadings on the factors $>0.50$ are in bold.

\begin{tabular}{|c|c|c|c|c|}
\hline Items (abbrev. content) & Calmness & Trainability & Dog sociability & Boldness \\
\hline Item19 (cool-headed) & 0.82 & 0.04 & 0.15 & -0.03 \\
\hline Item 15 (emotionally balanced) & 0.79 & 0.06 & 0.16 & 0.04 \\
\hline Item3 (calm) & 0.78 & -0.01 & 0.11 & -0.07 \\
\hline Item23 (anxious) ${ }^{\mathrm{a}}$ & 0.73 & 0.07 & 0.05 & 0.33 \\
\hline Item7 (stressed) $)^{\mathrm{a}}$ & 0.71 & 0.05 & 0.18 & 0.22 \\
\hline Item9 (intelligent) & 0.10 & 0.72 & 0.03 & -0.14 \\
\hline Item17 (dull) $)^{\mathrm{a}}$ & 0.16 & 0.71 & 0.01 & -0.01 \\
\hline Item13 (playful) & -0.04 & 0.68 & 0.07 & 0.23 \\
\hline Item 1 (inventive) & 0.06 & 0.64 & -0.04 & 0.06 \\
\hline Item21 (lazy) ${ }^{\mathrm{a}}$ & -0.10 & 0.62 & 0.13 & 0.17 \\
\hline Item24 (friendly) & 0.19 & 0.08 & 0.82 & 0.01 \\
\hline Item4 (quarrelling) ${ }^{\mathrm{a}}$ & 0.15 & 0.02 & 0.81 & -0.08 \\
\hline Item20 (bullying) ${ }^{\mathrm{a}}$ & 0.09 & 0.06 & 0.76 & 0.19 \\
\hline Item8 (kind) & 0.10 & 0.02 & 0.54 & -0.09 \\
\hline Item 10 (reserved) $^{\mathrm{a}}$ & -.08 & 0.19 & 0.04 & 0.77 \\
\hline Item 14 (aloof) ${ }^{\mathrm{a}}$ & 0.12 & -0.05 & 0.00 & 0.71 \\
\hline Item 18 (fearful) ${ }^{a}$ & 0.32 & 0.15 & -0.09 & 0.70 \\
\hline Explained variance & $23.81 \%$ & $13.86 \%$ & $11.41 \%$ & $8.60 \%$ \\
\hline Cronbach alpha & 0.85 & 0.71 & 0.75 & 0.65 \\
\hline Eigenvalues & 4.05 & 2.35 & 1.94 & 1.46 \\
\hline
\end{tabular}

a Scoring was reversed.

\subsection{Interactions between the personality factors and independent variables}

\subsubsection{Calmness}

Fig. 1 shows the regression tree model predicting calmness. Age, age at acquisition, neutering and training experience had the most significant effect on the calmness factor. The cross-validation method separated the whole sample into four subgroups by age $\left(F=89.72_{3,10515}, p<0.001\right)$. Dogs older than 6.9 years were subdivided by the age at acquisition $\left(F=8.88_{1,2177}, p<0.001\right)$. Dogs acquired before the age of 12 weeks were reported to have the highest mean calmness in the sample. This node consisted $10.2 \%$ of the dogs.

The 1-2.5-year-old and 2.5-4.8-year-old subgroups were subdivided by neutered status $\left(F=81.86_{1,4318}, p<0.001 ; F=36.30_{1,2668}\right.$, $p<0.001$, respectively). Unaltered dogs had higher mean calmness in both subgroups. Dogs with the lowest mean calmness were neutered and less than 2.5 years old. This node consisted of $13.6 \%$ of the sample.
The node of unaltered dogs younger than 2.5 years were further divided; the split was according to the dog's training experience $\left(F=14.54_{1,2875}, p<0.001\right)$. Dogs without any or with one type of training courses (e.g. guarding, agility) had lower calmness score.

In short, older dogs were calmer than their younger counterparts, and neutering was related to less calmness. Earlier acquisition of the dog was reported to enhance its calmness.

According to the GLM analysis, every independent variable had an effect on the calmness trait $\left(F=10.63_{78}, p<0.001\right)$. Below we list those effects that were not presented on the regression tree.

3.3.1.1. Main effects. Owner gender: Men had calmer dogs than women $\left(F=9.59_{1}, p<0.001\right)$.

Owner age: Owners under the age of 18 reported to have calmer dogs than others. 19-30-year-old owners had less calm dogs than others $\left(F=8.57_{3}, p<0.001\right)$.

Other dogs in the household: More dogs were related to higher calmness $\left(F=4.24_{3}, p<0.01\right)$.

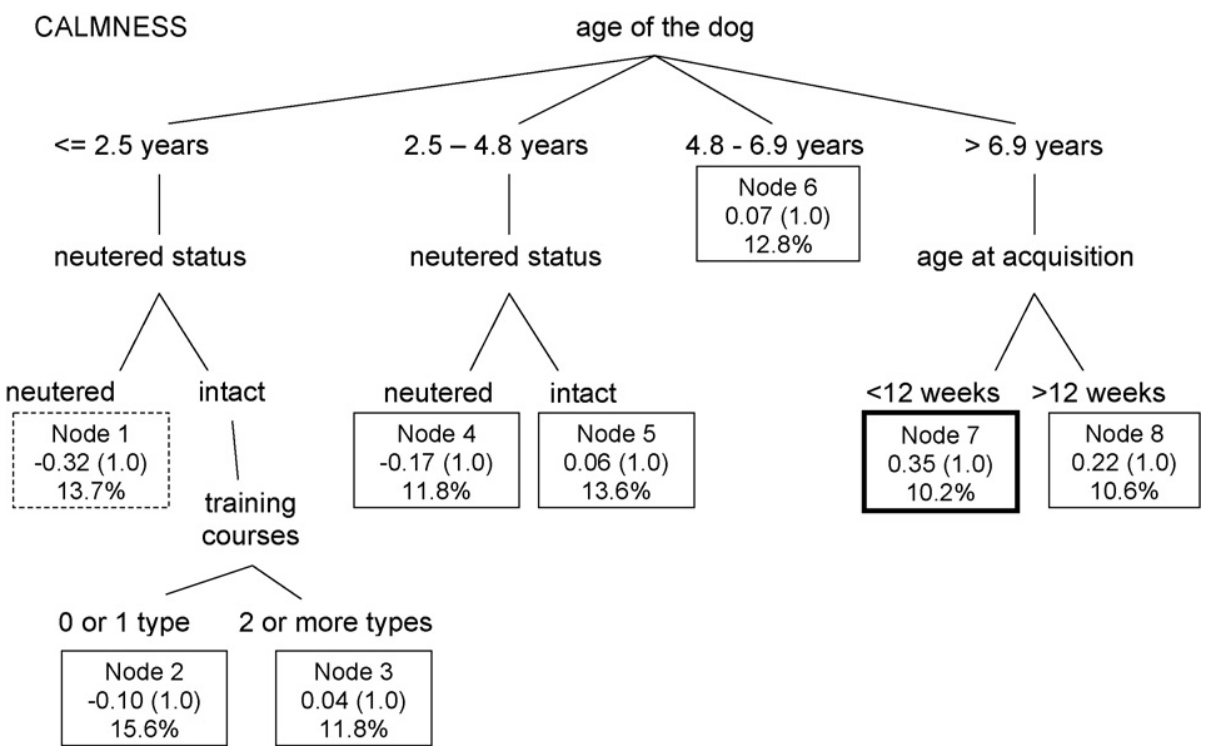

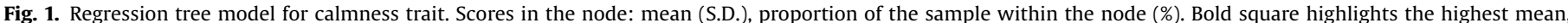
dashed line the lowest mean. 
3.3.1.2. Interactions. Neutered status and age: Calmness positively correlated with the age of the dogs in both neutered and intact dogs. However, the correlation was stronger in neutered dogs $\left(F=19.03_{1}\right.$, $p<0.001$ ).

Sex and neutered status: Intact dogs were reported to be calmer than their neutered counterparts in both sexes. However the difference was less significant in case of females than in case of males $\left(F=3.94_{1}, p<0.05\right)$.

Sex of the dog and number of people in the household: In female dogs the number of people was positively correlated with the calmness score: more people around was related to higher calmness. However, in male dogs there was no correlation $\left(F=11.81_{1}\right.$, $p<0.001$ ).

Neutered status and frequency of playing with the dog per week: Daily playing was related to a significantly higher calmness in intact dogs, however, this difference disappeared in case of the neutered dogs $(F=3.963, p<0.05)$.

Age at acquisition and education: Higher calmness was detected at dogs bred by the owner. In case the dog was acquired after its first birthday, secondary and high school educated owners had calmer dogs than primary-school and university educated owners $(F=2.029, p<0.05)$.Age at acquisition and age: Calmness positively correlated with the age in every 'age at acquisition' groups, except in the 'bred by the owner' group, at which there was no correlation $\left(F=3.39_{3}, p<0.05\right)$.

Age at acquisition and owner age: Children had the calmest dogs. Owners above the age of 60 years had generally less calm dog than others. However, in case of other owners, those, who acquired their dogs before the age of 12 weeks, reported moderately higher calmness compared to a delayed acquisition $\left(F=1.89_{9}, p<0.05\right)$.

Purpose of keeping the dogs and hours spent together: Calmness increased with the longer time the owner and the dog spend together. However, among owners, who claimed that their dog has other function besides being the member of the family, the reported calmness did not differ between the dogs (since it was relatively high primarily, $F=2.83_{4}, p<0.05$ ).

Purpose of keeping the dog and owner age: Owners above the age of 60 years had less calm dogs in case they kept their dog as family member only $\left(F=2.46_{6}, p<0.05\right)$.

Owner age and training experience: Dogs with at least two types of training experience were generally calmer than others, except in the group of owners under 18 and above 60 years $\left(F=2.18_{12}\right.$, $p<0.05)$.

\subsection{Trainability}

The regression tree model for predicting the trainability of dogs is illustrated in Fig. 2. Number of professional training courses the dogs has received (e.g. puppy class, obedience, agility), the age of the dog and purpose of keeping the dog were detected to have the most significant effect on the trainability score.

The first split was predicated on training experience $\left(F=220.13_{3,10515}\right)$. The subgroup of dogs who attended at least three types of professional training courses had the highest mean trainability. This group consisted of $19.8 \%$ of the sample.

The group of dogs without professional training experience was further split into two child nodes based on the age of the dog $\left(F=123.88_{1,3707}\right)$. Untrained dogs which were around or older than 3 years, had the lowest mean trainability in the sample. This node consisted of $17.2 \%$ of the dogs.

Dogs in the one type of training experience group generally attended basic obedience courses (puppy class and basic class). Their group was divided to two terminal nodes based on the purpose of keeping the $\operatorname{dog}\left(F=9.10_{1,2463}, p<0.01\right)$. Dogs described as the member of the family without any special purpose had lower mean scores on trainability than those dogs that had a more specific function in addition (e.g. work, guarding, etc.).

The two types of training experience group, similarly to the untrained dog-group, was split based the age of the dog $\left(F=40.19_{1,2259}, p<0.001\right) .2 .5$-year-old or younger than 2.5 -yearold dogs were reported to be more trainable than older dogs with similar types of training experience.

In short, according to the regression tree, the most important factor related to the trainability is the training experience of the dog. Additionally, younger dogs were generally reported to be more trainable than older dogs.

The GLM analysis revealed that every independent variables had detectable effects on the trainability variable $\left(F=17.30_{111}\right.$, $p<0.001)$. Below we list those main effects and interactions that are not represented on the regression tree of Fig. 2.

\subsubsection{Main effects}

Number of people in the household: More people around the dogs were related to less trainability $\left(F=5.03_{1}, p<0.01\right)$.

Purpose of keeping the dog: Dogs were reported to be more trainable in case their owner attributed specific function to the dog (work, guarding, etc.) not only being a family member $\left(F=13.70_{2}\right.$, $p<0.001$ ).

Playing per week and hours spent together: Frequent playing was related to higher trainability. However, those owners who played only once with their dog weekly reported higher level of trainability than it could be expected compared to others $\left(F=14.44_{3}\right.$, $p<0.001$ ).

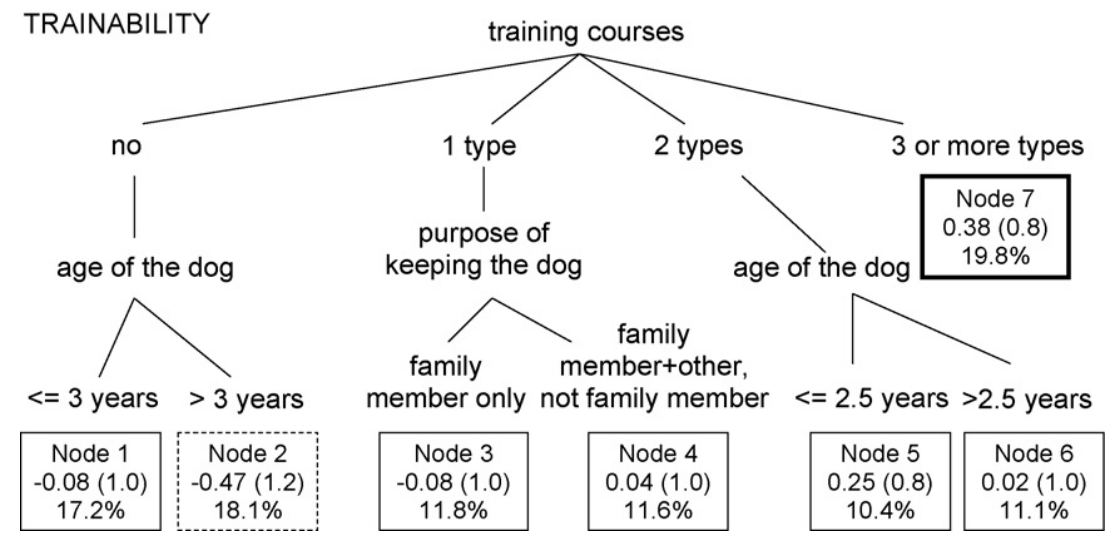

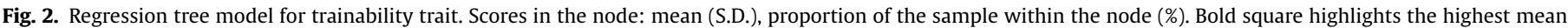
dashed line the lowest mean. 


\subsubsection{Interactions}

Sex and neutering: Neutered females and intact males were reported to be more trainable compared to neutered males and intact females $\left(F=4.76_{1}, p<0.05\right)$.

Neutering and the age of the dog: In the group of neutered dogs the negative correlation between the trainability and the age of the dogs was stronger than in the group of unaltered dogs $\left(F=9.23_{1}\right.$, $p<0.01$ ).

Age at acquisition and owner gender: Women had more trainable dogs than men, except if they acquired the dog after its first birthday $\left(F=3.01_{3}, p<0.05\right)$.

Age at acquisition and number of other dogs: Trainability decreased with the delayed acquisition of the dog. Dogs acquired after their first birthday and kept alone were reported to be the least trainable $\left(F=2.33_{9}, p<0.05\right)$.

Neutering and owner age: Trainability of neutered dogs slightly decreased with the age of the owners. However, in case of intact dogs, 19-30- and >60-year-old owners reported lower trainability $\left(F=2.99_{3}, p<0.05\right)$.

Training experience and age of the dog: Age negatively correlated with trainability in every 'training experience' group, but the correlation was less strong in case of more trained dogs $\left(F=2.84_{4}\right.$, $p<0.01$ ).

Age of the dog and hours spent together: The negative correlation between the age of the dog and the trainability score was the strongest in the group of dogs which spent less than $1 \mathrm{~h}$ with their owner daily $\left(F=7.59_{2}, p<0.001\right)$.

\subsubsection{Dog sociability}

The regression tree of dog sociability factor can be seen in Fig. 3. The first split on the sample was determined by the age of the $\operatorname{dog}\left(F=195.69_{4,10514}, p<0.001\right)$. Five age groups were formed. The youngest group (dogs under or around the one and a half year) had the highest sociability toward other dogs. The oldest age group (dogs above 4.8 years) was divided into subgroups by the hours spent together with the owner daily $\left(F=24.06_{1,3527}, p<0.001\right)$. The least sociable dogs were older than 4.8 years and spent less than $3 \mathrm{~h}$ together with the owner. Dogs who spent more than $3 \mathrm{~h}$ with the owners were subdivided again, by the sex of the $\operatorname{dog}\left(F=20.37_{1,2437}\right.$, $p<0.001$ ). Females were found to be more sociable toward their conspecifics than males.
According to the GLM, every independent variable had effect on the dog sociability factor $\left(F=12.19_{81}, p<0.001\right)$. Below we list those results that cannot be observed on the regression tree.

3.4.2.1. Main effects. Education of owner: Owners who had basic school education only reported the lowest dog-sociality, while owners with a university degree the highest. Secondary and high school educated owners were in between, at a similar level $\left(F=4.38_{3}, p<0.01\right)$.

Purpose of keeping the dog: Non-family member dogs were less sociable than family member dogs $\left(F=7.24_{2}, p<0.001\right)$.

Number of people in the household: Owners from more populated households reported having less dog-sociable $\operatorname{dog}\left(F=23.68_{1}\right.$, $p<0.001$ ).

Frequency of playing with the dog: More frequent playing was related to higher dog sociability $\left(F=6.26_{3}, p<0.001\right)$.

3.4.2.2. Interactions. Acquisition of the dog and gender of the owner: Delayed acquisition of the dog was related to lower sociability in both genders of owners. However, dogs of men, bred by the owner or acquired after the age of first year had the lowest mean of sociability $\left(F=3.40_{3}, p<0.05\right)$.

The number of professional training courses does not affect the sociability of the dog toward other dogs if they were acquired between the age of 2 weeks and 12 months. However, dogs that were bred by the owner and received one-two or four or more training courses were reported as less sociable. Dogs acquired after the age of 1 year and with three types of training experience were also found to be less sociable than the others $\left(F=1.96_{12}, p<0.05\right)$.

Neutered dogs who live with at least with two other dogs were the least sociable compared to others. Intact dogs had a mid-level of sociability except for those who live with at least two other dogs. These dogs were the most sociable compared to the whole population $\left(F=4.87_{3}, p<0.01\right)$.

\subsubsection{Boldness}

Fig. 4 presents the regression tree model of boldness. The sex of the dog, age at acquisition, and age of the dog had the strongest predictivity on the boldness factor. The first split was related to the dogs' sex $\left(F=196.59_{1,10517}, p<0.001\right)$. Males were divided into three subgroups $\left(F=28.97_{2,5898}, p<0.001\right)$ and females into two sub-

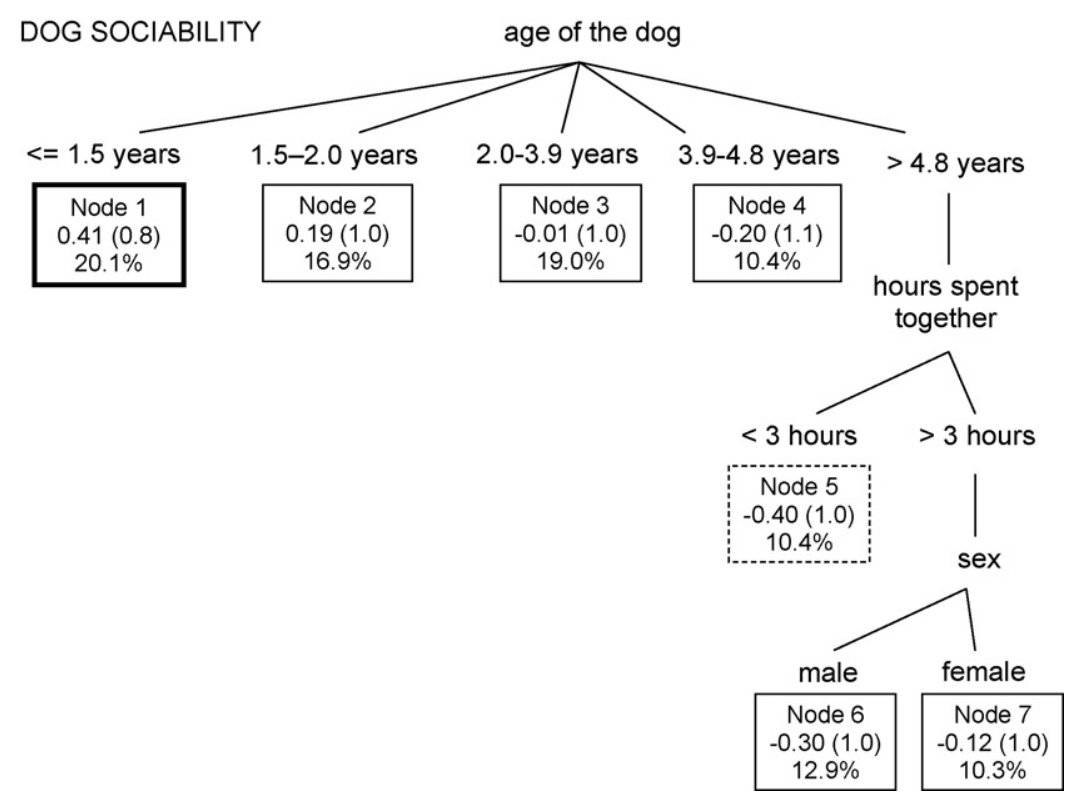

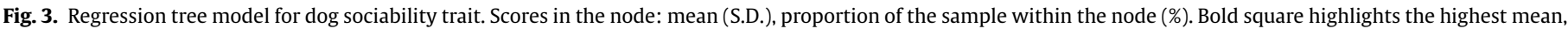
dashed line the lowest mean. 


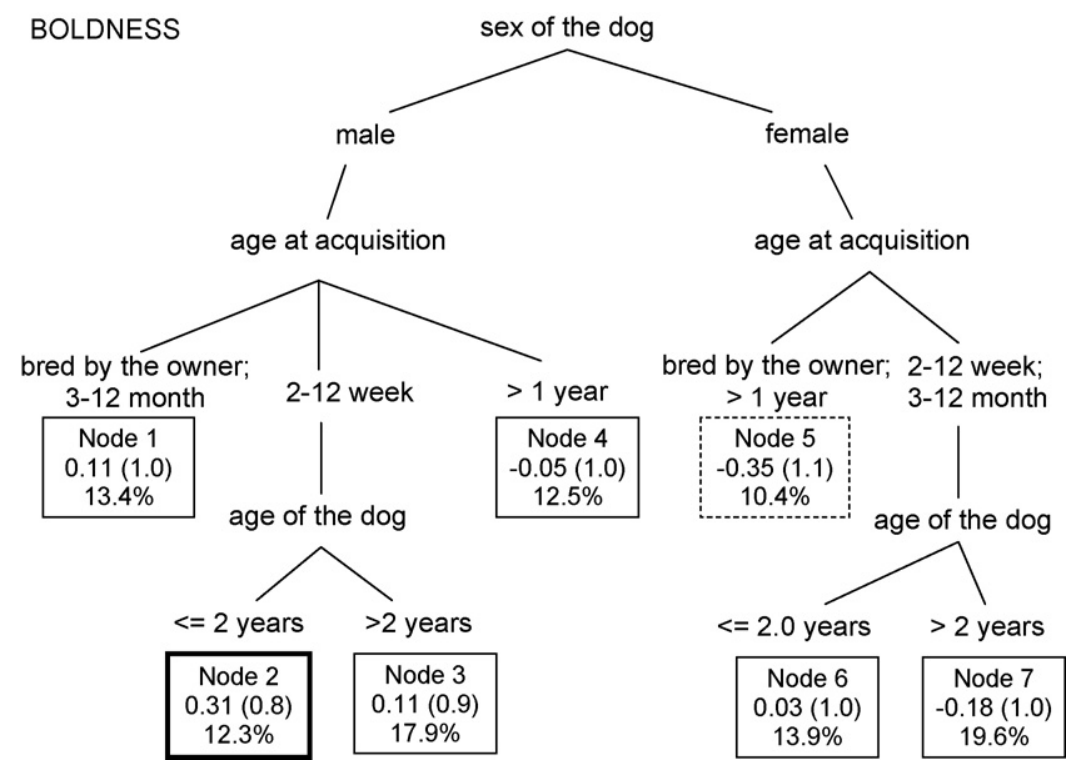

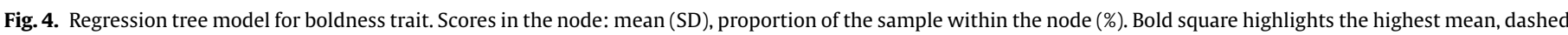
line the lowest mean.

groups $\left(F=51.23_{2,4616}, p<0.001\right)$ based on their age at acquisition. Two out of these five child nodes (see Fig. 4) were further subdivided by the dogs' ages ( $2-12$ weeks in males node: $\left(F=39.51_{1,3179}\right.$, $p<0.001$ and 2 weeks to 12 months in females node $\left.F=34.59_{1,3526}\right)$. The boldest dogs were males, acquired before the age of 12 weeks, and were younger than 2 years old ( $12.3 \%$ of the sample). The least bold dogs were females either acquired after the age of 1 year or bred by the owner ( $10.4 \%$ of the sample).

In short, younger dogs were more bold in both sexes. Females were outstandingly fearful if they were either bred by the owner or acquired after the age of their first year.

According to the GLM analysis 11 independent variables had effect on the fear factor $\left(F=10.73_{49}, p<0.001\right)$.

3.4.3.1. Main effects. Owner gender: Women's dogs were reported to be less bold $\left(F=6.93_{1}, p<0.01\right)$.

Number of other dogs: Single dogs were the boldest, while dogs who shared the household with two other dogs were reported to be the least bold $\left(F=3.16_{3}, p<0.05\right)$.

3.4.3.2. Interactions. Age at acquisition and age of the dog: Among dogs who were acquired between the age of 2-12 weeks, boldness negatively correlated with the age of the dog. In other groups there was no correlation $\left(F=6.61_{3}, p<0.001\right)$.

Training experience and age of the dog: Among dogs who attended 0-2 types of professional training courses, age negatively correlated with boldness: older dogs were more fearful. However, in dogs with three or more training courses there was no correlation $\left(F=3.74_{4}, p<0.01\right)$.Neutered status and age at acquisition: Among dogs acquired after the dog's first birthday intact individuals were reported to be bolder than neutered dogs $\left(F=3.40_{3}, p<0.05\right)$.

Neutered status and hours spent together: Among dogs who spend less than $3 \mathrm{~h}$ with their owner daily, neutered dogs were reported to be bolder. However, this trend has changed in the group of dogs who spend more than $3 \mathrm{~h}$ with their dog daily: in this case neutered dogs were less bold than their unaltered conspecifics. Boldness decreased with the longer time the dogs spend together with their owners in both groups $\left(F=3.37_{2}, p<0.05\right)$.

Purpose of keeping the dog and number of previous dogs: In dogs who were kept as family members exclusively, more experienced owners had less bold dog. In the groups of dogs who were kept for other purposes as well, there were no correlations $\left(F=4.06_{2}\right.$, $p<0.05$ ).

Sex of the dogs and number of previous dogs: In male dogs, more experienced owners had less bold dogs. In female dogs there was no correlation $\left(F=6.74_{1}, p<0.01\right)$.

Age of owner and training experience: Dogs of 19-30-year-old owners had bolder dogs than 31-60-year-old owners, except in the case of dogs who attended four or more training courses. Here the trend was opposite $\left(F=2.15_{12}, p<0.05\right)$.

\section{Discussion}

In the present questionnaire-based study with more than 14,000 respondents we identified four dog personality traits: calmness, trainability, dog sociability and boldness.

The four traits and their converses were described previously by several authors. Jones and Gosling (2005) conducted a metaanalysis on 51 empirical studies that were published before 2004 and extrapolated seven personality traits: reactivity, fearfulness, activity, sociability, responsiveness to training, submissiveness and aggression. Our four traits fit satisfactorily into Jones and Gosling's framework. Their reactivity trait is similar to our calmness trait, and our boldness trait is the converse of their fearfulness trait. While the meta-analysis of Jones and Gosling (2005) covered all areas of dog personality, our study did not touch upon activity, submissiveness and aggression in dogs, although some items in our sociability trait were aggression-related and there is some debate whether activity and submission should be considered a separate or independent personality traits (Gosling and John, 1999; Jones and Gosling, 2005).

The review of Jones and Gosling (2005) covered all types of temperament assessment methods and merged the results of the test batteries, ratings of individual dogs, expert ratings of breeds and observational tests. As Gosling argued elsewhere (Gosling, 2001), owner's ratings of individual dogs are as adequate as behavioral observations in case the ratings' validity and reliability were proved to be appropriate. Our personality traits in the questionnaire showed convincing repeatability and internal consistency. Our constructs had similar associations with independent variables as has been previously reported, which supports convergent validity (see below). 
Although working out a new tool (e.g. a questionnaire in this case) for collecting information about dogs' behavior in the home environment might be useful, the more important aspect of this study arises from the multiple demographic questions collected about both dogs and their owners. These variables allowed an extensive study on demographic and trait associations, some of them are new to the literature.

We used two statistical methods for investigating the associations. The more traditional univariate general linear models tested linear relationships and two-way interactions, however, due to the large number of independent variables, the numerous interactions are difficult to interpret. Regression trees are ideal for analyzing complex numeric and/or categorical data and detecting non-linear relationships in the structure (Breiman et al., 1984). As far as we know, this method has not been used to analyze large data sets in personality research on dogs, despite the fact that the method shows some advantages over other statistical approaches.

Below we summarize which independent variable had the most significant effect on our personality constructs and then we list the variables one by one for setting them in the literature. Note that the characteristics of dogs in the present study were reported by the owners, and the association with other traits or factors do not necessarily represent a causal relationship.

The results showed that calmness is influenced primarily by the dog's age, the neutered status, training experience and the age of acquisition. The least calm dogs were less than 2.5 years old, neutered and acquired after the first 12 weeks of age, while the calmest dogs were older than 6.9 years. Neutered dogs of either sex were less calm than their intact counterparts. More daily interaction and more experience with previous dogs on the owner's part was related to higher calmness. These findings are in accordance with the literature (Bennett and Rohlf, 2007; Wilsson and Sundgren, 1997; Kobelt et al., 2003) which support the convergent validity of this trait. Trainability is affected primarily by the number of types of professional training courses the dog received, the dog's age, and the purpose of keeping the dog. The least trainable dogs had not received professional training courses at all and were older than 3 years. The most trainable dogs were those who participated in three or more types of professional training courses. Daily interactions were also crucial for trainability (see Ley and Bennett, 2008; Bennett and Rohlf, 2007; Jagoe and Serpell, 1996; Kobelt et al., 2003). Dog sociability was mainly determined by the age, sex, training experience and time spent together. The least sociable dogs were older than 4.8 years and the owners spent less than $3 \mathrm{~h}$ with the dog daily. The most sociable dogs were less than 1.5 years old. Males were less sociable toward their conspecifics than females. Higher number of people in the household was related to less sociability too (see Bennett and Rohlf, 2007). Boldness was affected by the sex and age of the dog, the age of acquisition. The least bold were females acquired after the age of 1 year or bred by the owner. The boldest dogs were males, acquired before the age of 12 weeks, and were younger than 2 years old. These results are in harmony with the findings of Goddard and Beilharz, 1983; Wilsson and Sundgren, 1997; Ruefenacht et al., 2002; Svartberg, 2002; Strandberg et al., 2005).

Regression trees showed which demographic factors are the most predictive for the described personality traits. However, other variables, including the owner's gender, age, education, previous experience with dogs, the number of people and dogs in the household, and purpose of keeping the dogs also had less significant, but detectable effects.

Several findings revealed in the present study resonate with earlier reports. The dog owners of this study reported that generally older dogs were calmer, less trainable, less social and less bold than younger dogs. This is in harmony with the findings of Bennett and Rohlf (2007) who showed, using questionnaires in a volunteer sample, that the age of the dog was positively associated with unfriendliness and negatively associated with anxious behaviors. In another questionnaire-based study Ley and Bennett (2008) found that extraversion negatively correlated with age, also using a questionnaire. In contrast with these results, Seksel et al. (1999) did not find any associations with age in a behavioral test battery, while Strandberg et al. (2005) observed higher boldness in older dogs.

Age of acquisition is commonly believed to affect the adult behavior of the dog, but scientific evidence is rare. Bennett and Rohlf (2007) even called this belief a misconception, as they did not find any associations between that variable and problematic behaviors. However, according to our results, this variable had a significant effect on all personality traits in this study: dogs acquired before the age of 12 weeks were described as being calmer, more trainable, more social and bolder than dogs acquired later, especially those acquired as adults. This finding could be explained from two different perspectives. On the one hand, owners who acquire a dog before the age of 12 weeks could be more caring and more likely to plan in advance by consulting relevant references on dog behavior. Since the work of Scott and Fuller (1965), the idea that a dog should be adopted before the age of 12 weeks has been widespread in the dog literature. However, as shown by these authors, dogs can be socialized much easier during the so-called sensitive period between 8 and 12 weeks of age, so that direct positive effects from relatively early interactions cannot be excluded. Importantly, Serpell and Jagoe (1995) reported a relationship between the age of acquisition and problem behaviors. These included increased fear of other dogs and of traffic and was interpreted as the result of the so-called "kennel syndrome" in which young dogs are not exposed early enough to a variety of social and non-social stimuli.

From both theoretical and practical points of view, the effect of sex in dogs on various personality traits could be of great importance. We found that neutered females and intact males were reported to be more trainable compared to neutered males and intact females, which suggests that owners decide to neuter their male dogs if they have encountered behavioral problems or disobedience (see Hart and Miller, 1985; Bradshaw et al., 1996; Ruefenacht et al., 2002; Notari and Goodwin, 2007 for similar results, and Seksel et al., 1999; Bennett and Rohlf, 2007, who did not find major differences between the trainability of males and females).

Neutered dogs were less calm in both sexes. Bennett and Rohlf (2007) reported similar findings: desexed dogs were considered to be more nervous than sexually intact dogs. Intact males were the boldest group. Similar findings were reported with reference to "nerve stability" based on the behavioral test batteries (Goddard and Beilharz, 1983; Wilsson and Sundgren, 1997; Ruefenacht et al., 2002). Neutered females were less bold than all other sex-groups. Females were more sociable than males independently from altered status, as expected from Notari and Goodwin (2007). However, we have to emphasize again that the associations do not imply causal relationships. Neutering could well be the consequence of having experienced a behavioral problem, not the reason for showing a particular trait (see also Guy et al., 2001a,b).

One-third of dogs in our sample did not receive any type of professional training courses, similarly to an Australian sample (Kobelt et al., 2003). Dogs without any professional training courses were less calm, less trainable and less sociable than trained dogs. Dogs that received more types of training courses (e.g. guarding, agility) had significantly higher scores for all three traits. Trained dogs were reported as being bolder in comparison to non-trained companions. This corresponds to results obtained by Svartberg (2002). He found that trained dogs tested in the Dog Mentality Assessment test were bolder than untrained companions. In parallel, in a questionnaire survey, both Bennett and Rohlf (2007) and Kobelt et al. (2003) reported that trained dogs were more obedient. 
Previously there were little data on the relationship between the gender of the owner and the dog's personality. Bennett and Rohlf (2007) found that men reported having more disobedient dogs. By comparing the opinions of 2146 men and 8372 women about the behavior of their adult dogs we found that women's dogs were more trainable, more sociable and less bold than men's dogs. However, neither of these findings show whether the difference is in the eyes of the beholder or indeed if interactions between dogs and humans might be influenced by human sex differences.

We have to note here, that women were considerably more frequent in our sample. This could be explained by assuming that women keep dogs more frequently than men, are more willing to fill in questionnaires, or use the Internet more frequently. However, the latter assumption might not be relevant because other authors who did not require their subjects to use the Internet for filling in questionnaires published very similar gender rates (e.g. $85 \%$ of respondents were women in Bennett and Rohlf, 2007).

Older participants in Bennett and Rohlf's study (2007) reported that their dogs were more likely to appear anxious. In our sample, people aged between 19 and 30 years reported having the least calm dogs. The most trainable and sociable dogs could be found in the 31-60-year-old owner-group. However, the boldness scores of 31-60-year-olds' dogs were lower than those of dogs with 19-30year-old owners.

We did not find previous data in connection with dog-owners' educational history and their dogs' behavior. In our case primaryschool educated owners reported having less trainable and less social dogs than others. People with university degrees judged their dogs to be more social dogs in comparison to secondary-school educated owners.

Number of people in the household is another variable that can influence the behavior of dogs but has received little attention so far. In an Australian sample, dogs from larger families were rated as more disobedient and more unfriendly/aggressive (Bennett and Rohlf, 2007). Based on our sample, we can confirm that dogs in larger families were reported being less social toward theirs conspecifics than dogs living in smaller families. In female dogs the number of people was positively correlated with the calmness score: more people around was related to higher calmness. Additionally, a higher number of people in the household was associated with significantly bolder dogs. One possible explanation could be that people in larger families, which usually have one or more children, show less care and devotion toward their dogs. This seems to be supported by the finding that families with infants and children express a low degree of attachment toward their pets (including dogs), and the opposite is true for single or divorced people (Albert and Bulcroft, 1987).

In a similar vein, people living without children are more devoted to their dogs according to Marinelli et al. (2007). Thus, the family size, and potentially, the quality and quantity of interaction between family members and dogs has an influence on the personality traits of dogs. This suggests that it would be advisable to take this variable into account in future studies.

According to Kobelt et al. (2003), the number of dogs in the household did not affect personality traits. We found that a higher number of dogs in the household is associated with higher calmness and trainability, but decreased boldness. The reason for this finding is not clear; however, dogs could provide social partners for each other in the absence of the owner and be less bold on their own.

The reason for living with a dog is a key factor in the human-dog relationship, and it might affect the dog's personality. Companionship is a common reason for acquiring a dog-approximately $80 \%$ of a UK sample reported this as the main motivation (Jagoe and Serpell, 1996). In a random sample from Australia, 52\% of owners reported that companionship was the reason for getting the dog, and 74\% said companionship was the main benefit of having a dog (Kobelt et al., 2003). Dogs chosen for companionship showed lower rates of competitive aggression than dogs acquired for protection, breeding or exercise (Jagoe and Serpell, 1996), in contrast Kobelt et al. (2003) did not find such associations. In our questionnaire, the categories for the function of the dogs were not exclusive, so perhaps it is not surprising that $93.3 \%$ of the 14,004 respondents marked the 'family member' category as the function of their dogs. This suggests that German speaking Western European residents' attitudes toward their dogs can be characterized as affection and sympathy, rather than as economic self-interest (Serpell, 2004). By surveying the adult dogs only, we found that people who consider their dogs as family members exclusively (45.1\%) have less calm, less trainable, but bolder dogs than dogs in the "family member and other" category (48.2\%). The results suggest that dogs kept without any specific functions (e.g. work, guarding) are not as well trained as dogs with these functions.

Owners' experience with previous dogs seems to be an important factor. Dogs belonging to first-time dog owners were more likely to show behavior problems (Kobelt et al., 2003). Experienced owners had calmer dogs (Bennett and Rohlf, 2007), and experience increased the trainability or working success of dogs (questionnaire surveys: Jagoe and Serpell, 1996; Kobelt et al., 2003; Bennett and Rohlf, 2007; Ley and Bennett, 2008; test battery: Svartberg, 2002). Boldness was also higher in dogs living with experienced owners (questionnaire: Jagoe and Serpell, 1996; test battery: Svartberg, 2002). Similar to previous findings, we found that owners who had two or more previous dogs reported having calmer dogs than those owners who had no previous dogs or only one previous dog. Experienced owners had also more trainable dogs. However, contrary to the findings of Jagoe and Serpell (1996) and Svartberg (2002), boldness of the dogs in our sample did not increase with multiple training courses.

It is not surprising that owners who spend more time together with their dogs report to have calmer, more trainable, more sociable and less bold individuals. Since more time together generally means that the dog is kept in the house or in a flat rather than in a garden or a kennel, the result suggests that housing conditions probably affect the investigated traits.

Dog owners who engaged in training activities reported that their dogs were less disobedient, less nervous and more friendly toward people and dogs (Jagoe and Serpell, 1996; Kobelt et al., 2003; Bennett and Rohlf, 2007). We found that people who played every day with their dog perceived their pet to be calmer, more trainable and more social than those who played less. Importantly, this could be interpreted in two ways. People may prefer to play with calmer, more sociable dogs, or dogs could become calmer and more sociable as a result of frequent play.

Despite the virtues of the study (sample size and multiple personality trait-demographic variables associations), it has its own limitations. First, respondents were interested in reading DOGS magazine and completing a personality questionnaire about their dog, which biased the sample population. Presumably, this is one main reason why the majority of the respondents were women, although other authors published similar gender rates (e.g. 85\% in Bennett and Rohlf, 2007), which suggests that significantly more women than men have dogs. Second, the demographic variables may certainly be related to each other in several ways that were not uncovered here.

In summary, this large sample of dog owners allowed us to uncover a few major factors that may act as environmental variables in influencing the development of dog personality. Some of these are in agreement with previous studies based on questionnaires or behavioral observations, while others have not previously been reported. We think that the value of such research is in providing hypotheses for future work, which then should be executed 
under more controlled conditions, including the careful selection of a representative sample and with more focus on using direct behavioral measures.

\section{Acknowledgements}

The authors are grateful to Jesko Wilke, journalist associated with the German DOGS Magazine, for his cooperation in developing the questionnaire and for making this study possible. We also thank DOGS Magazine for its contribution in collecting data for us and for supporting the publication of these findings. Krisztina Nagy and Péter Kabai from the Institute for Biology at Szent István University helped in analyzing the data with regression trees. Micheal Grewe and the Canis Ltd. (Germany) helped us in collecting data for testing the reliability of the questionnaire. Two anonymous referees helped to improve the quality of the manuscript. We thank Michele Wan from the Department of Psychology at Columbia University for correcting the English in the manuscript. The research was funded by the FP7-ICT-2007 (LIREC-215554) and the Hungarian Scientific Research Fund (PD 48495).

\section{References}

Albert, A., Bulcroft, K., 1987. Pets and urban life. Anthrozöos 1, 9-23.

Bennett, P.C., Rohlf, V.I., 2007. Owner-companion dog interactions: relationships between demographic variables, potentially problematic behaviours, training engagement and shared activities. Appl. Anim. Behav. Sci. 102, 65-84.

Bradshaw, J.W.S., Goodwin, D., Lea, A.M., Whitehead, S.L., 1996. A survey of the behavioural characteristics of pure-bred dogs in the United Kingdom. Vet. Rec. 138, 465-468.

Breiman, L., Friedman, J.H., Olshen, R.A., Stone, C.G., 1984. Classification and Regression Trees. Wadsworth International Group, Belmont, CA, USA.

Cattell, R.B., 1966. The scree test for the number of factors. Multivariate Behav. Res. $1,245-276$.

De'Ath, G., Fabricius, K.E., 2000. Classification and regression trees: a powerful yet simple technique for ecological data analysis. Ecology 81, 3178-3192.

DeVellis, R.F., 1991. Scale Development: Theory and Applications. Sage Publications, Newbury Park, CA.

Diederich, C., Giffroy, J.M., 2006. Behavioural testing in dogs: a review of methodology in search for standardisation. Appl. Anim. Behav. Sci. 97, 51-72.

Goddard, M.F., Beilharz, R.G., 1983. Genetics of traits which determine the stability of dogs as guide-dogs for the blind. Appl. Anim. Ethol. 9, 299-315.

Gosling, S.D., 2001. From mice to men: what can we learn about personality from animal research? Psychol. Bull. 127, 45-86.

Gosling, S.D., John, O.P., 1999. Personality dimensions in non-human animals: a cross-species review. Curr. Dir. Psychol. Sci. 8, 69-75.

Guy, N.C., Luescher, U.A., Dohoo, S.E., Spangler, E., Miller, J.B., Dohoo, I.R., Bate, L.A., 2001a. A case series of biting dogs: characteristics of the dogs, their behaviour, and their victims. Appl. Anim. Behav. Sci. 74, 43-57.

Guy, N.C., Luescher, U.A., Dohoo, S.E., Spangler, E., Miller, J.B., Dohoo, I.R., Bate, L.A., $2001 \mathrm{~b}$. Risk factor for dog bites to owner in a general veterinary caseload. Appl. Anim. Behav. Sci. 74, 29-42.

Hart, B.L., Miller, M.F., 1985. Behavioral profile of dog breeds. JAVMA 186, 11751180.

Hsu, Y., Serpell, J.A., 2003. Development and validation of a questionnaire for measuring behavior and temperament traits in pet dogs. JAVMA 223, 12931300.

Jagoe, A., Serpell, J., 1996. Owner characteristics and interactions and the prevalence of canine behaviour problems. Appl. Anim. Behav. Sci. 47, 31-42.
Jones, A.C., Gosling, S.D., 2005. Temperament and personality in dogs (Canis familiaris): a review and evaluation of past research. Appl. Anim. Behav. Sci. 95, $1-53$.

Karels, T.J., Bryant, A., Hik, D.A., 2004. Comparison of discriminant function and classification tree analyses for age classification of marmots. Oikos 105, 575-587.

Kass, G., 1980. An exploratory technique for investigating large quantities of categorical data. Appl. Stats. 29, 119-127.

Kline, P., 1994. An easy guide to factor analysis. London: Routledge.

Kobelt, A.J., Hemsworth, P.H., Barnett, J.L., Coleman, G.J., 2003. A survey of dog ownership in suburban Australia-conditions and behaviour problems. Appl. Anim. Behav. Sci. 82, 137-148.

Ley, J., Bennett, P., 2008. Measuring personality in dogs. J. Vet. Behav. 3, 182

Ley, J., Bennett, P., Coleman, G., 2008. Personality dimensions that emerge in companion canines. Appl. Anim. Behav. Sci. 110, 305-317.

Low, M., Joy, M.K., Makan, T., 2006. Using regression trees to predict male provisioning of offspring in the stitchbird (hihi). Anim. Behav. 71, 1057-1068.

Marinelli, L., Adamelli, S., Normando, S., Bono, G., 2007. Quality of life of the pet dog: influence of owner and dog's characteristics. Appl. Anim. Behav. Sci. 108, 143-156.

Notari, L., Goodwin, D., 2007. A survey of behavioural characteristics of pure-bred dogs in Italy. Appl. Anim. Behav. Sci. 103, 118-130.

Pervin, L., John, O.P., 1997. Personality: Theory and Research, 7th ed. Wiley, New York, NY.

Podberscek, A.L., Serpell, J.A., 1996. The English Cocker Spaniel: preliminary findings on aggressive behaviour. Appl. Anim. Behav. Sci. 47, 75-89.

Rooney, N., Bradshaw, J.W.S., 2004. Breed and sex differences in the behavioural attributes of specialist search dogs-a questionnaire survey of trainers and handlers. Appl. Anim. Behav. Sci. 86, 123-135.

Ruefenacht, S., Gebhardt-Henrich, S., Miyake, T., Gaillard, C., 2002. A behaviour test on German Shepherd dogs: heritability of seven different traits. Appl. Anim. Behav. Sci. 79, 113-132.

Saetre, P., Strandberg, E., Sundgren, P.-E., Pettersson, U., Jazin, E., Bergström, T.F., 2006 The genetic contribution to canine personality. Genes Brain Behav. 5, 240-248.

Scott, J.P., Fuller, J.L., 1965. Genetics and the Social Behaviour of the Dog. University of Chicago Press, Chicago.

Seksel, K., Mazurski, E.J., Taylor, A., 1999. Puppy socialisation programs: short and long term behavioural effects. Appl. Anim. Behav. Sci. 62, 335-349.

Serpell, J.A., 2004. Factors influencing human attitudes to animals and their welfare. Anim. Welfare 13, S145-151.

Serpell, J., Jagoe, J.A., 1995. Early experience and the development of behavior. In: Serpell, J. (Ed.), The Domestic Dog. Cambridge University Press, pp. 80-102.

Sheppard, G., Mills, D.S., 2002. The development of a psychometric scale for the evaluation of the emotional predispositions of pet dogs. J. Comp. Psychol. 15 201-222.

Slabbert, J.M., Odendaal, J.S.J., 1999. Early prediction of adult police dog efficiency-a longitudinal study. Appl. Anim. Behav. Sci. 64, 269-288.

Strandberg, E., Jacobsson, J., Saetre, P., 2005. Direct genetic, maternal and litter effects on behaviour in German shepherd dogs in Sweden. Livest. Prod. Sci. 93, 33-42.

Svartberg, K., 2002. Shyness-boldness predicts performance in working dogs. Appl. Anim. Behav. Sci. 79, 157-174.

Svartberg, K., 2006. Breed-typical behaviour in dogs-historical remnants or recent constructs? Appl. Anim. Behav. Sci. 96, 293-313.

Svartberg, K., Forkman, B., 2002. Personality traits in the domestic dog (Canis familiaris). Appl. Anim. Behav. Sci. 79, 133-155.

van Oers, K., de Jong, G., van Noordwijk, A.J., Kempenaers, B., Drent, P.J., 2005. Contribution of genetics to the study of animal personalities: a review of case studies. Behaviour 142, 1191-1212.

Wilsson, E., Sundgren, P.-E., 1997. The use of a behaviour test for selection of dogs for service and breeding. I. Method of testing and evaluating test results in the adult dog, demands on different kinds of service dogs, sex and breed differences. Appl. Anim. Behav. Sci. 53, 279-295.

Wilsson, E., Sundgren, P.-E., 1998. Behaviour test for eight-week old puppiesheritabilities of tested behaviour traits and its correspondence to later behaviour. Appl. Anim. Behav. Sci. 53, 279-295.

Yamauchi, K., Ono, Y., Baba, K., Ikegami, N., 2001. The actual process of rating the Global Assessment of Functioning Scale. Compr. Psychiatry 42, 403-409. 